\title{
$\langle 100\rangle$-textured self-assembled square-shaped polycrystalline silicon grains by multiple shot excimer laser crystallization
}

\author{
Ming He, Ryoichi Ishihara, ${ }^{\text {a) }}$ Wim Metselaar, and Kees Beenakker \\ Laboratory of Electronic Components, Technology, and Materials (ECTM), Delft Institute of Microelectronics \\ and Submicron Technology (DIMES), Delft University of Technology, Feldmannweg 17, P.O. Box 5053, \\ 2600 GB Delft, The Netherlands
}

(Received 18 May 2006; accepted 18 July 2006; published online 18 October 2006)

\begin{abstract}
Strong preference for $\langle 100\rangle$ surface and in-plane orientations has been observed in polycrystalline silicon film on $\mathrm{SiO}_{2}$ after crystallization with multiple excimer laser pulses. Laser induced periodic surface structure (LIPSS) is developed in the film, constructing self-assembled square-shaped grains. The clear texture can be observed in a relatively wide energy density window, from 250 to $275 \mathrm{~mJ} / \mathrm{cm}^{2}$, for a $30 \mathrm{~nm}$ thick $\alpha$-Si layer. It is speculated that the lateral growth velocity of $\langle 100\rangle$-oriented grains is the fastest, and the orthogonal in-plane $\langle 100\rangle$ directions are developed due to the alternate directions of melting and solidification during the LIPSS formation. (C) 2006 American Institute of Physics. [DOI: 10.1063/1.2358405]
\end{abstract}

\section{INTRODUCTION}

Excimer laser crystallization (ELC) of $\alpha$-Si films is a well-established method to obtain polycrystalline silicon (poly-Si) grains, in which thin film transistors (TFTs) for large-area electronics can be fabricated. ${ }^{1}$ By twodimensional location control of grains, ${ }^{2}$ a TFT can be fabricated within a single crystalline grain and this $c$-Si TFT has superior performance. ${ }^{3}$ However, the device performance and the uniformity can be improved further by control of the crystallographic orientation of the channel of the TFTs. It is well known that electronic properties of metal oxide semiconductor field effect transistors ${ }^{4,5}$ (MOSFETs) have a pronounced dependence on the surface and in-plane crystal orientations with respect to the direction of the current flow due to the anisotropy of the effective mass. If the surface and even the in-plane orientations in the channel of the TFTs can be controlled in the location controlled grain, this grain will be ideal for $c$-Si TFT fabrication.

Kuriyama et al. ${ }^{6}$ have reported that poly-Si has a strong $\langle 111\rangle$ fiber texture after multiple shot ELC. Recently, Gosain et $a .^{7}{ }^{7}$ observed $\langle 100\rangle$ fiber texture within square-shaped grains after multiple shot ELC. However, in-plane orientations of the poly-Si grains have never been controlled. In this study, strongly preferred $\langle 100\rangle$ in-plane orientations are successfully obtained as well as the $\langle 100\rangle$ preferred surface orientation in square-shaped poly-Si grains by multiple shot excimer laser crystallization. Laser induced periodic surface structure (LIPSS) is observed and could be the reason for self-assembled square-shaped grains with three-dimensional texture.

\section{EXPERIMENT DETAILS}

A $30 \mathrm{~nm}$ thick $\alpha$-Si layer is deposited on thermally oxidized $\mathrm{Si}$ wafer $\left(1 \mu \mathrm{m}\right.$ thick $\left.\mathrm{SiO}_{2}\right)$ in a conventional horizontal hot-wall low pressure chemical vapor deposition

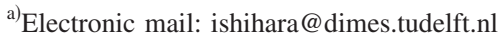

(LPCVD) reactor using pure silane as a source gas at a pressure of $20 \mathrm{~Pa}$ and a temperature of $547^{\circ} \mathrm{C}$. Subsequently, the $\alpha$-Si layer is irradiated with an excimer laser in a vacuum chamber by the XMR 5121 laser system $[\mathrm{XeCl}$ laser, $\lambda$ $=308 \mathrm{~nm}, \mathrm{FWHM}=50 \mathrm{~ns}$ (FWHM denotes full width at half maximum)] with an energy density varying from 250 to $280 \mathrm{~mJ} / \mathrm{cm}^{2}$ in $5 \mathrm{~mJ} / \mathrm{cm}^{2}$ steps. This energy density of the laser is slightly below the superlateral growth (SLG) region. ${ }^{8}$ The number of shots varies from 100 to 500, with a frequency of $5 \mathrm{~Hz}$. There is about $3^{\circ}$ between the incidence light and the normal direction of the surface. No intentional heating of the substrate is applied during the laser irradiation. Within the light path, a tilted quartz attenuator is used to reduce the energy density of the laser and to partly polarize the light.

\section{RESULTS AND DISCUSSIONS}

After excimer laser crystallization and Secco etching, morphologies of grains are investigated by scanning electron microscopy (SEM). Figure 1(a) is a SEM image showing the morphology of the poly-Si grains. Parallel, spatially periodic grain boundaries are visible and are well aligned in one direction with a uniform periodicity of about $300 \mathrm{~nm}$. With a high-resolution view [Fig. 1(b)], it is clearly shown that grains are nearly square shaped, with a grain size of $300 \mathrm{~nm}$, comparatively equal to the wavelength of excimer laser $(\lambda)$. It is found that the periodicity is independent of the number of shots. These characteristics indicate a very close connection to LIPSS. Kaki and Horita ${ }^{9}$ have reported that periodic grain boundaries were created during multiple shots solidstate laser crystallization of an $\alpha$-Si layer with linearly polarized light. Although excimer laser light is known to have a minor degree of polarization, in the current experiment settings, the tilted quartz attenuator makes the light partly polarized.

The LIPSS has been confirmed by atomic force microscopy (AFM) analysis. Figure 2 shows the AFM image of 5 $\times 5 \mu \mathrm{m}^{2}$ area of poly-Si grains. The neighboring grains form 


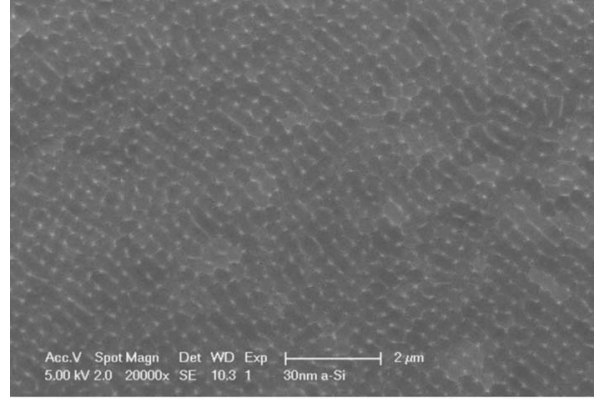

(a)

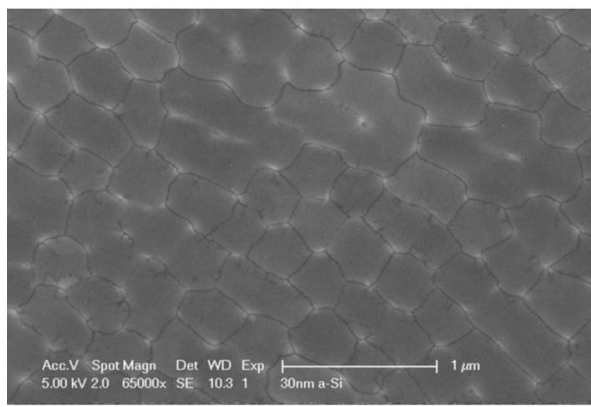

(b)

FIG. 1. SEM images of poly-Si grains crystallized at $260 \mathrm{~mJ} / \mathrm{cm}^{2}$ after 500 shots: (a) periodic grain boundaries and (b) self-assembled square-shaped grains.

a hillock, which represents the grain boundary. The periodic grain boundaries are aligned on one line, with a uniform spacing of about $300 \mathrm{~nm}$. Square-shaped grains are selfassembled and form four hillocks at each corner of the square. Root-mean-square (rms) roughness of the surface is $15 \mathrm{~nm}$, which is in the same region of poly-Si crystallized by a nonpolarized laser light.

Figure 3 shows the pole figures and inverse pole figure of poly-Si grains, analyzed by electron backscatter diffraction (EBSD) with an automatic mapping method. The clear preference of $\langle 100\rangle$ texture is visible not only in the surface orientation [Fig. 3(b)] but also in the in-plane orientation [Fig. 3(a)]. This preferred in-plane orientation is achieved for lateral growth of $\mathrm{Si}$ grains. Furthermore, the preferred inplane $\langle 100\rangle$ orientation is either parallel or perpendicular to the LIPSS direction, i.e., always perpendicular to the sides of the square. The prominent texture component ${ }^{10}$ is $\{001\}\langle 110\rangle$. About $43 \%$ of preferred in-plane orientation is estimated over the other in-plane crystallographic orientations, within a $10^{\circ}$ misorientation tolerance.

The aforementioned strongly textured and selfassembled square-shaped grains are obtained in a relatively wide laser energy density window. In Fig. 4 the energy density window is plotted. For a fixed number of pulses, the energy density window is approximately $10 \mathrm{~mJ} / \mathrm{cm}^{2}$. By lowering the energy density or the number of shots, no LIPSS is developed. On the other hand, above the window, the agglomeration or ablation of the film occurs.

LIPSS formation on the surfaces of semiconductors ( $\mathrm{Si}$ and $\mathrm{Ge}$ ) and metal ( $\mathrm{Al}$ and $\mathrm{Cu}$ ) has been intensively investigated. ${ }^{11}$ Spatially periodic temperature profile, due to the interference of incoming or refracted laser beams and the light scattered by the surface, has been speculated as the cause for LIPSS formation. ${ }^{12}$ Furthermore, at a low fluence, LIPSS can be developed by solidification of periodically and locally molten strips, which was confirmed by time-resolved diffraction. ${ }^{13}$ The energy density applied in the experiment is slightly below the threshold for the uniform melting of $\mathrm{Si}$ layer; therefore the periodic local melting could be the reason for LIPSS formation. Figure 5 shows schematically the pulse-to-pulse alternate directions of melting and solidification when LIPSS is fully developed. After the $(n-1)$ th shot ( $n$ is an integer), periodic grain boundaries are formed at positions of $m \lambda$ ( $m$ is an integer). In the $n$th shot, temperatures in the valleys of LIPSS are higher than those at the hillocks because of the preferential energy accumulation of laser light in the valley ${ }^{9,14}$ and the effectively thick Si layer at the hillocks. The modulated temperature profile is out of phase with the preformed LIPSS. The melting starts from the valley to the hillocks and stops at the middle between the valleys and leaves some unmolten $\mathrm{Si}$. The solidification starts from these unmolten $\mathrm{Si}$ and solidification directions are opposite to the melting directions. It then forms hillocks (grain boundaries) at position of $\left(m+\frac{1}{2}\right) \lambda$ due to the collision of two solidification fronts. That is, the location of the grain

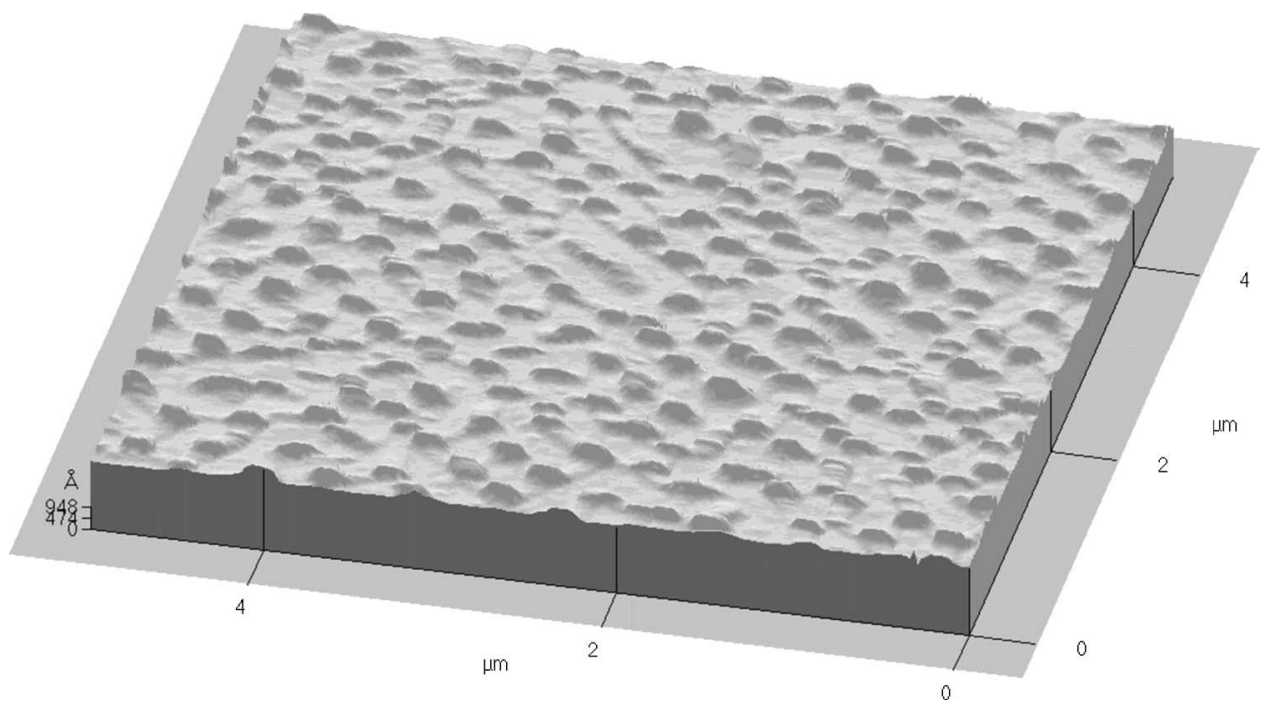

FIG. 2. AFM image of the poly-Si grains crystallized at $260 \mathrm{~mJ} / \mathrm{cm}^{2}$ after 500 shots. 

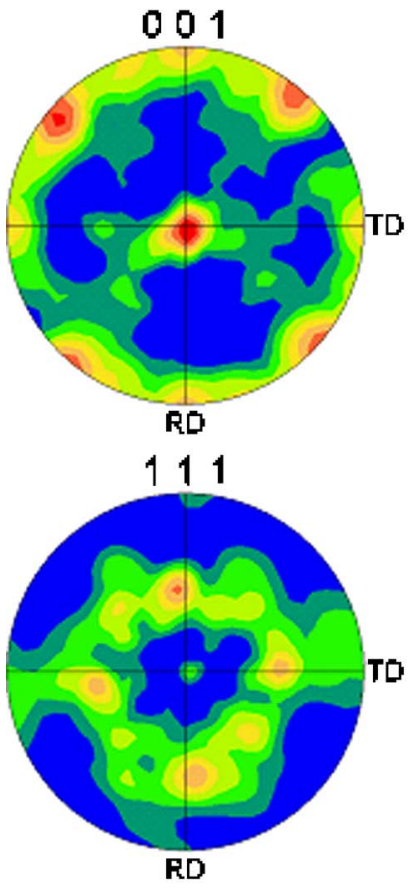

(a)

boundary changes on a shot-to-shot basis. Similarly, after additional shot $(n+1)$ th, grain boundaries return to the original positions $m \lambda$. Consequently, the grain boundary location moves forward and backward in the directions normal to the LIPSS on two successive shots. As a result, the poly-Si is self-assembly shaped into squares with hundreds of meltingsolidification cycles.

The $\langle 100\rangle$ texture has been explained by the anisotropy of the melting temperature. ${ }^{7}$ It is argued that $\langle 100\rangle$-oriented grains have a higher melting temperature and can coexist in

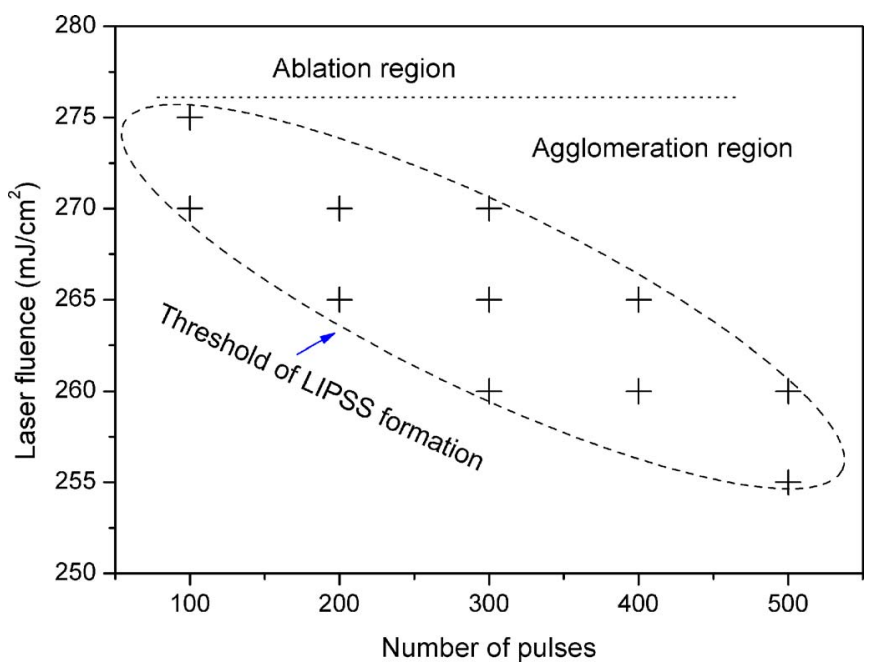

FIG. 4. (Color online) The energy density window to obtain the textured self-assembled poly-Si grains. The cross points indicate the experimental points to get the textured self-assembled poly-Si grains. The dashed line indicates laser energy density window combined with the number of shots. Above the dotted line, is the ablation region. In between, it is the agglomeration region.

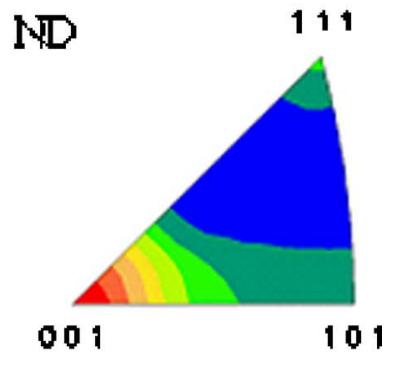

FIG. 3. (Color online) The texture of poly-Si grains crystallized at $260 \mathrm{~mJ} / \mathrm{cm}^{2}$ after 500 shots: (a) Pole figure shows the $\langle 100\rangle$ surface orientation and $\langle 100\rangle$ in-plane orientations, (b) Inverse pole figure of normal direction (ND), which shows the strong $\langle 100\rangle$ surface orientation. molten Si liquid, as a result of which the new solidified poly-Si grains have $\langle 100\rangle$-preferred orientation. However, the difference of the melting temperature is negligibly small. For a $0.5 \mu \mathrm{m}$ thick film, it is about $10^{-2} \mathrm{~K}$. At the melting temperature, the heat conductivity of solid or molten $\mathrm{Si}$ is high enough to annihilate the small difference. In this study (Fig. 4), the $\langle 100\rangle$ texture can be obtained within a relatively wide energy density window. Furthermore the liquid-solid coexistence is reported to be stable only when the Si contacts with oxide on both sides. ${ }^{15}$ Another explanation for the preferred orientation is the anisotropy of surface free energy, ${ }^{16}$ which is argued for the strong $\langle 111\rangle$ texture obtained by multiple shot ELC. ${ }^{6}$ However, the above anisotropy of the melting temperature is caused by and calculated from the anisotropy of the surface free energy. ${ }^{15}$ Thus by the same reason, it could be possible to obtain either $\langle 111\rangle$ or $\langle 100\rangle$ texture after the multiple shot ELC.

In this study, the strongly preferred $\langle 100\rangle$ orientation could be developed by the alternate directions of the solidification. The AFM image (Fig. 2) shows there are hillocks at the four corners of each square-shaped grain. Figure 5 only indicates the alternate directions of solidification perpendicular to the LIPSS. The alternate directions of solidification also occur parallel to the LIPSS, due to the periodic hills and valleys. Thus melting-solidification cycle requires in-plane fourfold symmetrical lateral growth direction, the bipolarities of the directions perpendicular and parallel to the LIPSS. It is suggested here that the growth rate, for a given undercooling, is the fastest for $\langle 100\rangle$ direction than other directions, analogous to the dendrite growth direction for cubic metals. ${ }^{17}$ As indicated in Fig. 4, LIPSS is not well developed until a certain number of shots for each proper energy level. The tex- 

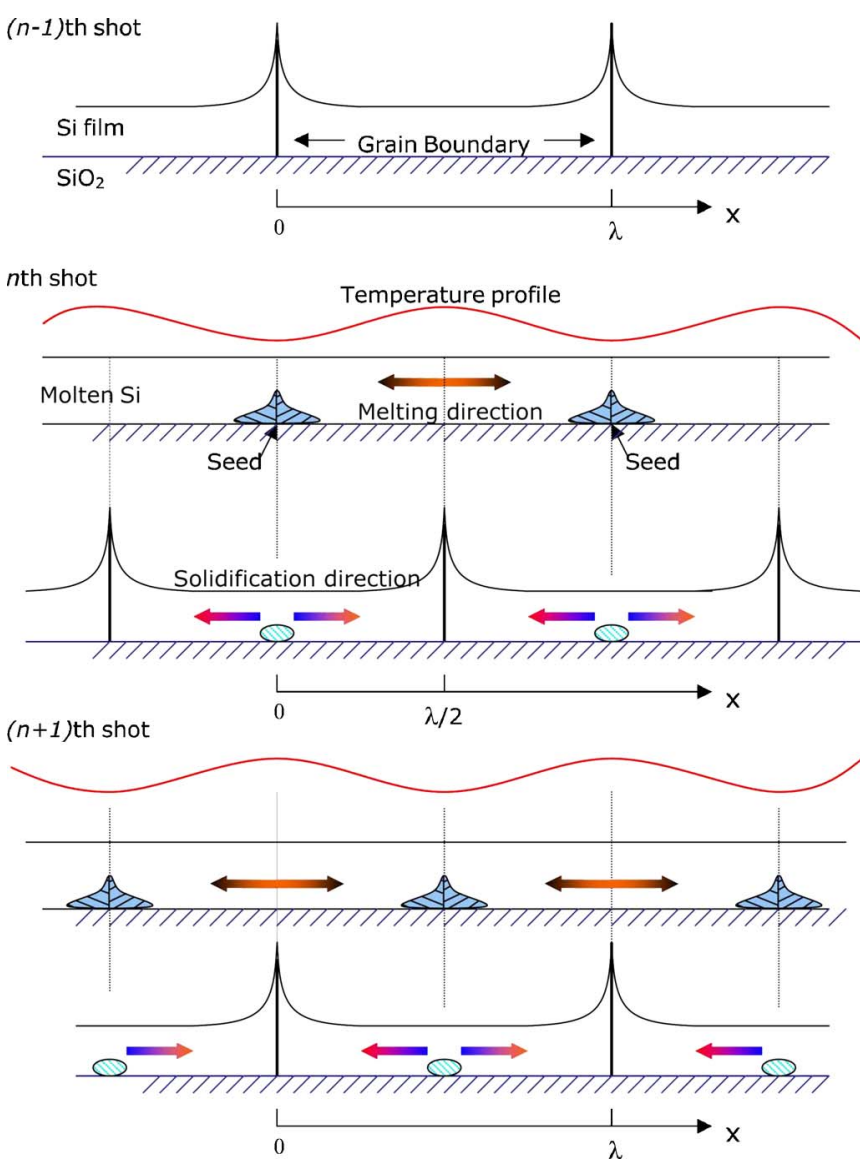

FIG. 5. (Color online) Schematic drawings of the periodic melting, alternate direction of the solidification and spatially alternate grain boundaries, after the LIPSS is fully developed. After $(n-1)$ th shot ( $n$ is an integer), the grain boundaries are at $m \lambda$ ( $m$ is an integer). At $n$th shot, due to the preferential accumulation of laser energy in the valleys, the melting starts from $\left(m+\frac{1}{2}\right) \lambda$, leaving some unmolten $\mathrm{Si}$ at $m \lambda$. The solidification of molten $\mathrm{Si}$ forms new grain boundaries at $\left(m+\frac{1}{2}\right) \lambda$. Subsequently for the $(n+1)$ th shot, the grain boundaries return to $m \lambda$.

ture is developed in the course of LIPSS formation. Figure 6 shows a schematic explanation for the $\langle 100\rangle$ texture during the development of LIPSS. After the initial $(k-1)$ th shot $(k$ is an integer), the crystal orientations of grains are random. A grain with a $\langle 100\rangle$ surface orientation and $\langle 100\rangle$ direction perpendicular to the LIPSS can grow to the largest grain. The grain boundary is formed outside $\lambda / 2$ as the grain with $\langle 100\rangle$ orientation grows fastest. Grains with other orientations cannot grow beyond the $\lambda / 2$. In the following $k$ th shot, melting starts from the valley $(\lambda / 2)$, and subsequently the hillocks are melted because their positions are near the hot valleys. The melting stops near the middle between the valleys and leaves the $\mathrm{Si}$ at 0 and $\lambda$ unmelted. These two unmolten crystals are single crystalline and function as $\langle 100\rangle$-oriented seeds during the solidification. After the solidification, two large grains are formed and the length of $\langle 100\rangle$ surface oriented crystals is doubled. It should be noted that the length of $\langle 100\rangle$ orientation increases also in the parallel direction to LIPSS. After the additional $(k+1)$ th shot, three seeds with $\langle 100\rangle$ orientation are formed at the positions of $\pm \lambda / 2$ and $\frac{3}{2} \lambda$. Although the seeds at $-\lambda / 2$ and $\frac{3}{2} \lambda$ are single crystalline, the seed at $+\lambda / 2$ could be two grains. However, since these two grains are originally from the same grain, they will be crys-

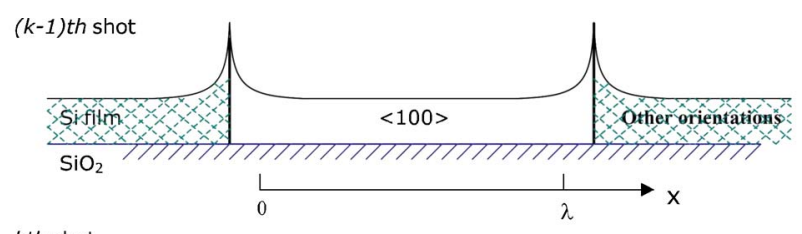

kth shot

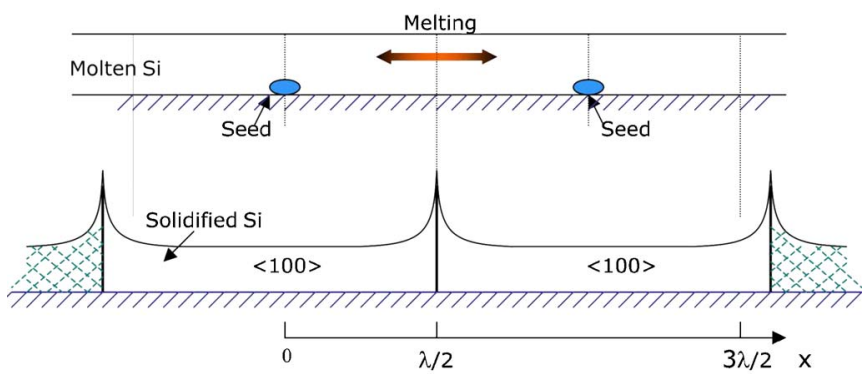

$(k+1)$ th shot

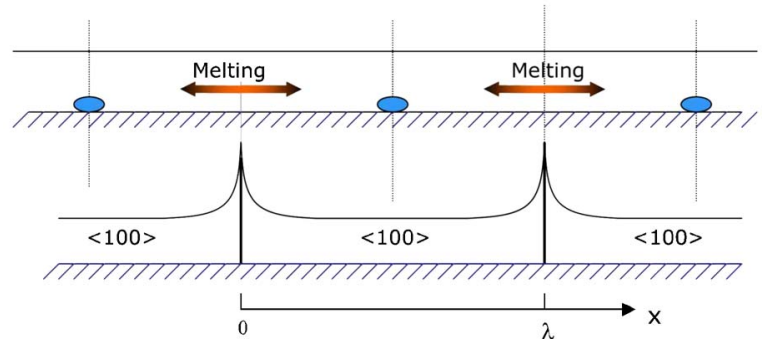

FIG. 6. (Color online) schematic explanation of the $\langle 100\rangle$ texture developing during the three successive shots while LIPSS is developing. After the $(k-1)$ th shot ( $k$ is an integer), one grain with $\langle 100\rangle$ orientation grows to be the largest grain. During the $k$ th shot, two seeds survived after the melting and hence the length of $\langle 100\rangle$ direction doubled. In the flowing $(k+1)$ th shot, three seeds were formed, as a result the length of $\langle 100\rangle$ direction tripled.

tallographically merged at a high temperature and eventually form a single crystal. When the melting-solidification cycle is repeated, the preferred $\langle 100\rangle$ orientation is "inherited" to outside, while other orientations are occluded by the growth of $\langle 100\rangle$-oriented grains. With hundreds of the meltingsolidification cycles, the in-plane preferred $\langle 100\rangle$ directions are perpendicular and parallel to the LIPSS [Fig. 3].

$\langle 100\rangle$-crystal orientation on $\mathrm{SiO}_{2}$ is the most desirable orientation for the manufacturing of complementary MOS (CMOS) integrated circuits (ICs) owing to lowest density of fixed oxide charge and interface electronic states. ${ }^{18}$ This $\langle 100\rangle$-textured film can be used for TFT fabrication and the TFT should have better characteristics. Furthermore, this $\langle 100\rangle$-textured film could be used as seeds, combined with location control, ${ }^{2}$ to prepare the orientation and location controlled grains for TFT fabrication or three-dimensional (3D) ICs.

\section{CONCLUSIONS}

In summary, the strongly $\langle 100\rangle$-textured self-assembled square-shaped poly-Si grains are prepared from $30 \mathrm{~nm} \alpha-\mathrm{Si}$ precursor by multiple shot ELC. The strong texture coexists with the LIPSS and can be obtained in a relatively wide energy density window. It is speculated that the $\langle 100\rangle$ orientation has the fastest growth rate. During the LIPSS formation, in-plane directions of solidification, perpendicular and 
parallel to the LIPSS, require in-plane fourfold symmetry and the $\langle 100\rangle$ orientation is selected and other orientations are occluded. This strong textured film can be used for TFT fabrication or seeds to prepare the orientation controlled grains.

\section{ACKNOWLEDGMENTS}

The authors would like to thank Ellen Neihof, Yvonne Andel, and John Slabbekoorn for technical help for ELC. The authors would like to give thanks to Dr. Yasushi Hiroshima and Macro Krogt for the EBSD and AFM analyses, respectively. This work was partly supported by the Dutch Foundational Research for Matters (FOM) (Project No. 97TF05).

${ }^{1}$ S. Uchikoga and N. Ibaraki, Thin Solid Films 383, 19 (2001).

${ }^{2}$ P. C. van der Wilt, B. D. van Dijk, G. J. Bertens, R. Ishihara, and C. I. M. Beenakker, Appl. Phys. Lett. 79, 1819 (2001).

${ }^{3}$ R. Ishihara et al., IEEE Trans. Electron Devices 51, 500 (2004).

${ }^{4}$ T. Sato, Y. Takeishi, H. Hara, and Y. Okamoto, Phys. Rev. B 4, 1950
(1971).

${ }^{5}$ T. Sato, Y. Takeishi, and H. Hara, Jpn. J. Appl. Phys. 8, 588 (1969).

${ }^{6}$ H. Kuriyama, T. Nohda, S. Ishida, T. Kuwahara, S. Noguchi, S. Kiyama, S. Tsuda, and S. Nakano, Jpn. J. Appl. Phys., Part 1 32, 6190 (1993)

${ }^{7}$ D. P. Gosain, A. Machida, T. Fujino, Y. Hitsuda, K. Nakano, and J. Sato, Jpn. J. Appl. Phys., Part 2 42, L135 (2003).

${ }^{8}$ J. S. Im and H. J. Kim, Appl. Phys. Lett. 64, 2303 (1994).

${ }^{9}$ H. Kaki and S. Horita, J. Appl. Phys. 97, 014904 (2005).

${ }^{10} \mathrm{~V}$. Randle, Microtexture Determination and its Applications, 2nd ed. (Maney, London, 2003).

${ }^{11}$ J. F. Young, J. S. Preston, H. M. van Driel, and J. E. Sipe, Phys. Rev. B 27, 1155 (1983).

${ }^{12}$ J. E. Sipe, J. F. Yong, J. S. Preston, and H. M. van Driel, Phys. Rev. B 27, 1141 (1983).

${ }^{13}$ J. F. Young, J. E. Sipe, and H. M. van Driel, Phys. Rev. B 30, 2001 (1984).

${ }^{14}$ T. D. Lee, H. W. Lee, C. H. Nam, J. K. Kim, and C. O. Park, J. Appl. Phys. 71, 4208 (1992).

${ }^{15}$ H. I. Smith, C. V. Thompson, M. W. Geis, R. A. Lemons, and M. A. Bosch, J. Electrochem. Soc. 130, 2050 (1983).

${ }^{16}$ R. J. Jacodin, J. Electrochem. Soc. 110, 524 (1963).

${ }^{17}$ B. Chalmers, Principles of Solidification (Wiley, New York, 1964).

${ }^{18}$ S. M. Sze, Semiconductor Devices: Physics and Technology, 2nd ed. (Wiley, New York, 2002). 